\title{
On the Symmetry of $b$-Functions of Linear Free Divisors
}

\author{
by
}

\author{
Michel Granger and Mathias Schulze
}

\begin{abstract}
We introduce the concept of a prehomogeneous determinant as a possibly nonreduced version of a linear free divisor. Both are special cases of prehomogeneous vector spaces. We show that the roots of the $b$-function are symmetric about -1 for reductive prehomogeneous determinants and for regular special linear free divisors. For general prehomogeneous determinants, we describe conditions under which this symmetry persists.

Combined with Kashiwara's theorem on the roots of $b$-functions, our symmetry result shows that -1 is the only integer root of the $b$-function. This gives a positive answer to a problem posed by Castro-Jiménez and Ucha-Enríquez in the above cases.

We study the condition of strong Euler homogeneity in terms of the action of the stabilizers on the normal spaces.

As an application of our results, we show that the logarithmic comparison theorem holds for reductive linear Koszul free divisors exactly when they are strongly Euler homogeneous.
\end{abstract}

2010 Mathematics Subject Classification: 14M17, 20G05, 17B66, 14F40.

Keywords: linear free divisor, prehomogeneous vector space, $b$-function.

\section{Contents}

$\S 1$ Logarithmic comparison theorem and $b$-functions 480

§2. Linear free divisors and prehomogeneous determinants 482

$\S 3$. b-functions of reductive prehomogeneous determinants 487

§4. Examples 491

$\S 5$. $b$-functions of special linear free divisors 498

$\S 6$. Euler homogeneity and normal representations 500

§7. Koszul freeness and Euler homogeneity 502

References 504

Communicated by M. Kashiwara. Received March 4, 2009

M. Granger: Département de Mathématiques, Université d'Angers, LAREMA, CNRS UMR $\mathrm{n}^{\mathrm{O}}$ 6093, 2 Bd Lavoisier, 49045 Angers, France;

e-mail: granger@univ-angers.fr

M. Schulze: Department of Mathematics, Oklahoma State University, Stillwater, OK 74078, USA; e-mail: mschulze@math.okstate.edu

(C) 2010 Research Institute for Mathematical Sciences, Kyoto University. All rights reserved. 


\section{$\S 1$. Logarithmic comparison theorem and $b$-functions}

Let $D$ be a reduced hypersurface in a complex $n$-dimensional manifold $X$, let $U=X \backslash D$, and let $j: U \hookrightarrow X$ be inclusion. Then the de Rham morphism

$$
\Omega_{X}^{\bullet}(* D) \rightarrow \mathbf{R} j_{*} \mathbb{C}_{U}
$$

is a quasi-isomorphism. This so-called Grothendieck's comparison theorem is the core of the proof of Grothendieck's algebraic de Rham theorem Gro66] in which $D$ plays the role of a divisor at infinity for a compactification. In particular, for Stein $X$, each cohomology class $c \in H^{k}(U ; \mathbb{C})$ is represented as $c(\sigma)=\int_{\sigma} \omega$ by a differential $k$-form $\omega$ with finite pole order along $D$. The natural question of limiting this pole order dates back to Griffiths Gri69] and has been studied later by Deligne and Dimca [DD90, Dim91], Karpishpan [Kar91, and others.

By analogy with Grothendieck's comparison theorem, one says that the logarithmic comparison theorem ( $L C T$ ) holds for $D$ if the inclusion

$$
\Omega_{X}^{\bullet}(\log D) \hookrightarrow \Omega_{X}^{\bullet}(* D)
$$

is a quasi-isomorphism. The explicit characterization of hypersurfaces for which the LCT holds is an open problem (see Tor07] for an overview). Mainly the extremal cases of isolated singularities and free divisors (as defined in [Sai80]) have been studied. For isolated singularities the LCT can be explicitly characterized in the quasihomogeneous case (see [HM98]) and there are partial results for the nonquasihomogeneous case (see [Sch07]). Also several variants of the LCT property have been introduced and studied. For instance, F. J. Calderón Moreno and L. Narváez Macarro [CN09] generalized the LCT to integrable logarithmic connections. More famous is a global algebraic version of the LCT which is conjectured to hold for all hyperplane arrangements in characteristic 0 (see [Ter78, Conj. 3.1] and [WY97, §1]).

In this article we shall be concerned with the case of free divisors. The main conjecture in this case is the following from [CMNCJ02, Conj. 1.4].

Conjecture 1.1. For a free divisor, the LCT implies strong Euler homogeneity.

By definition, strong Euler homogeneity at $p$ for some $p \in D$ means that $f \in \mathfrak{m}_{X, p} \cdot J(f)$ for some, and hence any, local defining equation $f \in \mathscr{O}_{X, p}$ at $p$, where $J(f)$ denotes the ideal of partials of $f$. By strong Euler homogeneity of $D$ without reference to the point we mean strong Euler homogeneity at all $p \in D$ (see Definition 6.1 for more details). We have proved Conjecture 1.1 for Koszul free divisors (see Section 7 for their definition) and for $n \leq 3$ in GS06, Cor. 1.5, Thm. 1.6]. For plane curves, F. J. Calderón-Moreno et al. [CMNCJ02, Thm. 3.5] 
showed that the implication in Conjecture 1.1 is even an equivalence, but for $n \geq 3$ a counter-example for the converse implication is missing. In fact the first result in this direction was a stronger sufficient condition for LCT, proved by F. J. Castro-Jiménez et al. CNM96, which appeared to be too strong to be necessary: Any locally quasihomogeneous free divisor satisfies the LCT. By definition, locally quasihomogeneous hypersurfaces admit at any point a defining equation which is quasihomogeneous with respect to strictly positive weights on a local coordinate system. As observed by L. Narváez Macarro [NM08, Rem. 1.7.4], the above implication still holds true even without this strictness requirement. The resulting condition is referred to as local weak quasihomogeneity. His argument is based on the following characterization of the LCT in terms of $\mathscr{D}_{X}(\log D)$-modules given in [CN05, Cor. 4.3].

Theorem 1.2. A free divisor $D$ satisfies the logarithmic comparison theorem if

(a) $\mathscr{D}_{X} \stackrel{L}{\otimes} \mathscr{D}_{X}(\log D) \mathscr{O}_{X}(D)$ is concentrated in degree 0 ,

(b) $\mathscr{D}_{X} \otimes_{\mathscr{D}_{X}(\log D)} \mathscr{O}_{X}(D) \rightarrow \mathscr{O}_{X}(* D)$ is injective.

Condition (a) is fulfilled for Koszul free $D$ and condition (b) is equivalent to $\operatorname{Ann}_{\mathscr{D}_{X}}\left(f^{-1}\right)$ being generated by order one operators. This latter condition is studied in Tor04 where the following is proved in Tor04, Cor. 1.8] for the Koszul free case.

Theorem 1.3. Let $f \in \mathscr{O}_{X}$ be a Koszul free germ on $X=\left(\mathbb{C}^{n}, 0\right)$. Then $\operatorname{Ann}_{\mathscr{D}_{X}}\left(f^{-1}\right)$ is generated by order one operators if and only if

(a) $f$ is Euler homogeneous,

(b) -1 is the only integer root of the (local) b-function of $f$,

(c) $f$ and the symbols of a basis of $\operatorname{Der}(-\log f)$ form a $\operatorname{gr}_{F} \mathscr{D}_{X}$-regular sequence.

A promising class of examples to make the preceding theorems more explicit in order to better understand the LCT problem is that of linear free divisors. These are free divisors $D$ in a complex vector space $X=V \cong \mathbb{C}^{n}$ such that $\operatorname{Der}(-\log D)$ admits a basis of vector fields with linear coefficients. Linear free divisors have been introduced in BM06] where they occur as discriminants in certain quiver representation spaces. Later they have been studied in GMNS09 and dGMS09. We have proved a global version of the LCT [GMNS09, Thm. 1.6] for reductive linear free divisors and it is conceivable that LCT holds for any linear free divisor.

The interest in linear free divisors arises from the fact that they are special cases of prehomogeneous vector spaces, which endows them with a rich additional structure that has been studied intensively. A particular feature in view of The- 
orem 1.3 is that their $b$-functions are much more explicit and satisfy, in certain cases, a functional equation due to M. Sato's fundamental theorem for prehomogeneous vector spaces. The present paper is motivated by the wish to understand $b$-functions of linear free divisors in order to apply Theorems 1.2 and 1.3 . It is organized as follows:

In Section 2, we introduce prehomogeneous determinants as a slight generalization of linear free divisors for which all our arguments work without additional effort. We further study reductivity and a condition that is referred to as special in dGMS09, Def. 2.1]. In Theorem 2.4 we give a more elementary proof of the implication "reductive implies special" from dGMS09, Cor. 2.9] for the more general case of prehomogeneous determinants. In Section 3, we study $b$-functions of prehomogeneous determinants and prove our main result in Theorem 3.5 which states that the roots of the $b$-function of any reductive prehomogeneous determinant are symmetric about -1 , which implies, in particular, that condition (b) of Theorem 1.3 holds true.

Theorem 1.4. The roots of the b-function of any reductive linear free divisor are symmetric about -1 .

In Section 4 , we compute the $b$-function for all linear free divisors in dimension up to 4, and, using the microlocal calculus from [SKKŌ81], for some prehomogeneous determinants arising from quiver theory. Based on this list of examples we expect that the symmetry property holds true for any linear free divisor.

Conjecture 1.5. The statement of Theorem 1.4 holds true also for nonreductive linear free divisors.

Sections 5 and 6 contain additional material independent of our application to the LCT: We show in Theorem 5.5 that the symmetry statement in our main result holds true also for regular special linear free divisors, where "regular" refers to the corresponding notion for prehomogeneous vector spaces (see [Kim03, Def. 2.14]). In Proposition 6.6, we give a sufficient condition for Euler homogeneity at a point in terms of the corresponding normal representation. In Section 7] we discuss condition (c) in Theorem 1.3 in order to finally prove the following statement on the LCT for linear free divisors.

Theorem 1.6. A reductive linear Koszul free divisor satisfies the logarithmic comparison theorem if and only if it is strongly Euler homogeneous. 


\section{$\S 2$. Linear free divisors and prehomogeneous determinants}

A reduced hypersurface $D$ in an $n$-dimensional complex vector space $V$ is called a linear free divisor if the module $\operatorname{Der}(-\log D)$ of logarithmic vector fields has a basis of global degree 0 vector fields

$$
\delta_{k}=A_{k} x=x^{t} A_{k}^{t} \partial_{x} \in \Gamma(V, \operatorname{Der}(-\log D))_{0}, \quad A_{k} \in \mathbb{C}^{n \times n}, \quad k=1, \ldots, n .
$$

Then, by Saito's criterion [Sai80, Thm. 1.8.ii],

$$
f=\operatorname{det}\left(\delta_{1}, \ldots, \delta_{n}\right) \in \operatorname{Sym}\left(V^{*}\right)
$$

is a homogeneous defining equation for $D$ of degree $n$. Therefore we can assume that $\delta_{1}=\epsilon$ is the Euler vector field

$$
\epsilon=\frac{1}{n} \sum_{i=1}^{n} x_{i} \partial_{x_{i}}
$$

and then also that $\delta_{2}, \ldots, \delta_{n} \in \Gamma(V, \operatorname{Der}(-\log f))_{0}$ where $\operatorname{Der}(-\log f)$ is the module of vector fields tangent to (the level sets of) $f$.

We denote by $G_{D} \subseteq \mathrm{GL}_{V}$ the unit component of the stabilizer of $f \in$ $\mathbb{P} \operatorname{Sym}\left(V^{*}\right)$, and by $A_{D} \subseteq G_{D}$ that of $f \in \operatorname{Sym}\left(V^{*}\right)$. The following was shown in GMNS09, Lemmas 2.2-2.4]: $G_{D}$ is a linear algebraic group with Lie algebra of infinitesimal generators $\mathfrak{g}_{D}=\Gamma(V, \operatorname{Der}(-\log D))_{0}$, and the same holds for $A_{D}$ with $\mathfrak{a}_{D}=\Gamma(V, \operatorname{Der}(-\log f))_{0}$. By Saito's criterion, $V \backslash D$ is a Zariski open orbit with finite isotropy groups and $G_{D}$ an $n$-dimensional group. Conversely, any reduced discriminant of an $n$-dimensional linear algebraic group in $\mathrm{GL}_{V}$ with an open orbit is a linear free divisor. Thus, linear free divisors are special cases of discriminants of prehomogeneous vector spaces $(G, \rho, V)$ characterized by the following conditions:

1. $G \subseteq \mathrm{GL}_{V}$ is connected with $\operatorname{dim} G=\operatorname{dim} V$,

2. $\rho=\mathrm{id}: G \hookrightarrow \mathrm{GL}_{V}$,

3. $f(x)=\operatorname{det}\left(d \rho\left(A_{1}\right) x, \ldots, d \rho\left(A_{n}\right) x\right) \in \operatorname{Sym}\left(V^{*}\right), \mathfrak{g}=\left\langle A_{1}, \ldots, A_{n}\right\rangle$, is reduced.

We shall consider the more general objects defined by dropping the third condition. However, we shall frequently impose the additional condition of reductivity on $G$ when we study $b$-functions in Section 3 .

Definition 2.1. Let $G \subseteq \mathrm{GL}_{V}$ be a connected algebraic group with $\operatorname{dim} G=$ $\operatorname{dim} V=n$ such that $(G, \rho, V)$ is a prehomogeneous vector space where $\rho=\mathrm{id}$ : $G \hookrightarrow \mathrm{GL}_{V}$. Consider the relative invariant

$$
f(x)=\operatorname{det}\left(d \rho\left(A_{1}\right) x, \ldots, d \rho\left(A_{n}\right) x\right) \in \operatorname{Sym}\left(V^{*}\right)
$$


defined by generators $A_{1}, \ldots, A_{n}$ of the Lie algebra $\mathfrak{g}$ of $G$. We call the (possibly nonreduced) discriminant divisor $D \subseteq V$ defined by $f$ a prehomogeneous (discriminant) determinant, and a linear free divisor in case $f$ is reduced. We call $D$ reductive if $G$ is a reductive group. We set $G_{D}=G, \mathfrak{g}_{D}=\mathfrak{g}$, and $\rho_{D}=\rho$, and we denote the character of $f$ by $\chi_{D}: G_{D} \rightarrow \mathbb{C}^{*}$ and its derivative by $d \chi_{D}: \mathfrak{g}_{D} \rightarrow \mathbb{C}$. We call

$$
A_{D}=\left(\operatorname{ker} \chi_{D}\right)^{\circ}, \quad \mathfrak{a}_{D}=\operatorname{ker} d \chi_{D},
$$

the annihilator of $D$ where ${ }^{\circ}$ denotes the unit component.

While $G_{D}$ is determined by $D$ if $D$ is a linear free divisor, it is part of the data of a general prehomogeneous determinant $D$. Note that, in Definition 2.1 . $f$ is indeed a relative invariant by [Kim03, Thm. 2.9]. Then $\chi_{D}$ is not trivial by [Kim03, Prop. 2.4(2)] and we have

$$
G_{D} / A_{D} \cong \mathbb{C}^{*}, \quad \mathfrak{g}_{D} / \mathfrak{a}_{D} \cong \mathbb{C} .
$$

In particular, we may pick $A_{1} \in \mathfrak{g}_{D}$ such that

$$
d \chi_{D}\left(A_{1}\right)=1 .
$$

We choose $A_{1}=E / n$ if $D$ is a linear free divisor. For $A \in \mathfrak{g}_{D}$, we abbreviate

$$
\delta_{A}=\left\langle d \rho_{D}(A) x, \partial_{x}\right\rangle=x^{t} A^{t} \partial_{x}, \quad \delta_{k}=\delta_{A_{k}} .
$$

By definition of a relative invariant [Kim03, §2.2] and [Kim03, Lem. 2.15],

$$
f \circ \rho_{D}(g)=\chi_{D}(g) \cdot f, \quad \delta_{A}(f)=\left\langle d \rho_{D}(A) x, \operatorname{grad}_{x}(f)\right\rangle=d \chi_{D}(A) \cdot f .
$$

As $\operatorname{deg} f=n$ by (2.1) and $G_{D} \subseteq \mathrm{GL}_{V}$ with $\operatorname{dim} V=n$ by Definition 2.1, we have

$$
\operatorname{deg} \chi_{D}=n=\operatorname{deg}\left(\operatorname{det} \circ \rho_{D}\right) .
$$

Lemma 2.2. For a prehomogeneous determinant $D, \chi_{D}=\operatorname{det} \circ \rho_{D}$ if and only if $\operatorname{tr} \circ d \rho_{D}=0$ on $\mathfrak{a}_{D}$.

Proof. The converse being trivial, let us assume that the second condition holds. As $d$ det $=$ tr, this gives using 2.2 that

$$
\operatorname{ker} d\left(\operatorname{det} \circ \rho_{D}\right) \supseteq \mathfrak{a}_{D}=\operatorname{ker} d \chi_{D}, \quad \operatorname{ker}\left(\operatorname{det} \circ \rho_{D}\right) \supseteq A_{D}=\left(\operatorname{ker} \chi_{D}\right)^{\circ} .
$$

Then both $\chi_{D}$ and det $\circ \rho_{D}$ induce characters on $G_{D} / A_{D}$. But the latter has character group isomorphic to $\mathbb{Z}$ by (2.3). Using (2.7), this implies the equality $\chi_{D}=\operatorname{det} \circ \rho_{D}$. 
Lemma 2.3. If $D$ is a linear free divisor or a reductive prehomogeneous determinant, one can choose $A_{1} \in \mathfrak{g}_{D}$ with (2.4) such that

$$
\mathfrak{g}_{D}=\mathbb{C} \cdot A_{1} \oplus \mathfrak{a}_{D}, \quad \mathfrak{a}_{D}=\left\langle A_{2}, \ldots, A_{n}\right\rangle .
$$

Proof. With $A_{1}=E / n$, the first statement follows from (2.1) and Saito's criterion. By Jac79, Ch. III, $\S 7$, Thm. 10], reductivity of $\mathfrak{g}_{D}$ means that

$$
\mathfrak{g}_{D}=\mathfrak{c}_{D} \oplus \mathfrak{s}_{D}
$$

where $\mathfrak{c}_{D}$ is the center of $\mathfrak{g}_{D}, d \rho_{D}\left(\mathfrak{c}_{D}\right)$ consists of semisimple endomorphisms, and $\mathfrak{s}_{D}$ is a semisimple ideal. By semisimplicity of $\mathfrak{s}_{D}$, we have

$$
\mathfrak{s}_{D}=\left[\mathfrak{s}_{D}, \mathfrak{s}_{D}\right]
$$

Then it follows from 2.2 that

$$
\mathfrak{s}_{D}=\left[\mathfrak{g}_{D}, \mathfrak{g}_{D}\right] \subseteq \mathfrak{a}_{D}
$$

and hence $A_{1} \notin \mathfrak{s}_{D}$ by (2.4). Using (2.9) and (2.11), this shows that $A_{1} \in \mathfrak{c}_{D}$ after subtracting a suitable element of $\mathfrak{s}_{D}$.

In order to say more, we shall focus on the reductive case for the remainder of this section. The following result is proved in dGMS09, Cor. 2.9] in the case of linear free divisors.

Theorem 2.4. Let $D$ be a reductive prehomogeneous determinant. Then

$$
\chi_{D}=\operatorname{det} \circ \rho_{D}, \quad d \chi_{D}=\operatorname{tr} \circ d \rho_{D},
$$

which means that $A_{D} \subseteq \mathrm{SL}_{V}$.

Proof. We resume the notation $\mathfrak{c}_{D}$ and $\mathfrak{s}_{D}$ from Lemma 2.3. By 2.11, $\mathfrak{s}_{D} \subseteq \mathfrak{a}_{D}$ and hence $\mathfrak{a}_{D}=\mathfrak{c}_{D}^{\prime} \oplus \mathfrak{s}_{D}$ where $\mathfrak{c}_{D}^{\prime}$ is the center of $\mathfrak{a}_{D}$.

Now let $\mathfrak{h}_{D}$ be a Cartan subalgebra of $\mathfrak{s}_{D}$. Recall that $A_{1} \in \mathfrak{c}_{D}$ by Lemma2.3. Pick generators $S_{1}=A_{1}, S_{2}, \ldots, S_{s}$ of $\mathfrak{c}_{D}+\mathfrak{h}_{D}$ such that $d \rho_{D}\left(S_{1}\right), \ldots, d \rho_{D}\left(S_{s}\right)$ are semisimple and extend them to a basis $N_{1}, \ldots, N_{r}, r=n-s$, of $\mathfrak{a}_{D}$ such that $N_{1}, \ldots, N_{r}$ are $\left(\mathfrak{c}_{D}+\mathfrak{h}_{D}\right)$-homogeneous and $d \rho_{D}\left(N_{1}\right), \ldots, d \rho_{D}\left(N_{r}\right)$ are nilpotent. Then, by (the proof of) GMNS09, Thm. 6.1(4)], we have

$$
d \chi_{D}\left(S_{i}\right)=\operatorname{tr} \circ d \rho_{D}\left(S_{i}\right)+\sum_{j=1}^{r}\left[S_{i}, N_{j}\right] / N_{j}, \quad i=1, \ldots, s .
$$

This implies that $d \chi_{D}=\operatorname{tr} \circ d \rho_{D}$ on $\mathfrak{c}_{D}$. But on $\mathfrak{s}_{D}$, both $d \chi_{D}$ and $\operatorname{tr} \circ d \rho_{D}$ are zero by 2.11) and 2.10. Now the claim follows from Lemma 2.2 
Our motivation to study the symmetry of $b$-functions of prehomogeneous determinants stems from M. Sato's fundamental theorem for prehomogeneous vector spaces [Kim03, Prop. 4.18]. We shall need the following result to show that it holds for reductive prehomogeneous determinants in Theorem 3.3 .

Theorem 2.5. Let $D$ be a reductive linear free divisor.

(a) The Lie algebra representation $\rho_{D}$ is defined over $\mathbb{Q}$ with respect to some $V_{\mathbb{Q}}$.

(b) With respect to any $V_{\mathbb{Q}}$ as in (a), also $D$ is defined over $\mathbb{Q}$.

(c) With respect to any $V_{\mathbb{Q}}$ as in (a), also $G_{D}$ is defined over $\mathbb{Q}$.

For the convenience of the reader we give a proof of the following general fact which is probably well-known to specialists.

Lemma 2.6. Every finite-dimensional representation $V$ of a complex semisimple Lie algebra $\mathfrak{s}$ is defined over $\mathbb{Q}$ with respect to some $V_{\mathbb{Q}}$.

Proof. Let $\mathfrak{h}$ be a Cartan and $\mathfrak{b}$ be a Borel subalgebra of $\mathfrak{s}$. By Chevalley's Normalization [Ser01, Ch. VI, $\S 6$, Thm. 11], $\mathfrak{s}$ has a rational form $\mathfrak{s} \mathbb{Q}$. By complete reducibility we may assume that $V$ is an irreducible $\mathfrak{s}$-module. Let $V_{\omega}=U(\mathfrak{s}) \otimes_{\mathfrak{b}} L_{\omega}$ where $U(\mathfrak{s})$ denotes the universal enveloping algebra of $\mathfrak{s}$ and $L_{\omega}$ is a complex onedimensional $\mathfrak{b}$-module of weight $\omega \in \mathfrak{h}^{*}$. Then set $E_{\omega}=V_{\omega} / N_{\omega}$ where $N_{\omega}$ is generated by all $\mathfrak{s}$-modules strictly contained in $V_{\omega}$. By [Ser01, Ch. VII, §3, Thm. 2], $V=E_{\omega}$ where $\omega$ is integer on the coroots in $\mathfrak{h}_{\mathbb{Q}}$ by [Ser01, Ch. VII, $\S 4$, Prop. 3(b)]. This implies that $V_{\omega}$ is a direct sum of rational weight spaces for $\mathfrak{h}_{\mathbb{Q}}$ and is thus defined over $\mathbb{Q}$. Then $N_{\omega}$ is the maximal $\mathfrak{s}$-module in $V_{\omega}$ that meets $L_{\omega}$ in zero. Choosing a $\mathbb{Q}$-basis of $L_{\omega}, V_{\omega}$ also decomposes as a direct sum of $\mathbb{Q}$-vector spaces $U\left(\mathfrak{s}_{\mathbb{Q}}\right) \otimes_{\mathfrak{b}_{\mathbb{Q}}} L_{\omega, \mathbb{Q}}$ compatible with the above direct sum decomposition. Clearly $N_{\omega}$ is homogeneous with respect to both direct sums and hence defined over $\mathbb{Q}$.

Proof of Theorem 2.5. We show that $\rho_{D}$ is defined over $\mathbb{Q}$ when restricted to $\mathfrak{a}_{D}$, which implies (a) by (2.8) and $A_{1}=E / n$. Then (b) follows by (2.1), and (c) as in the proof of GMNS09, Lem. 2.2].

We resume the notation $\mathfrak{c}_{D}^{\prime}, \mathfrak{s}_{D}$, and $\mathfrak{h}_{D}$ from the proof of Theorem 2.4 As $\mathfrak{c}_{D}^{\prime} \oplus \mathfrak{h}_{D}$ is commutative, its representation on $V$ can be diagonalized. By Lemma 2.6, the representation of $\mathfrak{s}_{D}$ on each $\mathfrak{c}_{D}^{\prime}$-weight space of $V$ is defined over $\mathbb{Q}$. So there is a $\mathbb{Q}$-subspace $V_{\mathbb{Q}}$ of $V$ with respect to which $\left.\rho_{D}\right|_{\mathfrak{s}_{D}}$ is defined over $\mathbb{Q}$ and with respect to which $\rho_{D}\left(\mathfrak{c}_{D}^{\prime} \oplus \mathfrak{h}_{D}\right)$ consists of semisimple endomorphisms (with possibly complex weights). As $\mathbb{C} \cdot A_{1} \oplus \mathfrak{c}_{D}^{\prime} \oplus \mathfrak{h}_{D}$ is a Cartan subalgebra of $\mathfrak{g}_{D}$, it follows from Ser01, Ch. III, $\S 5$, Thm. 3(b)] that it equals its centralizer. Then [Sai71, Lem. 1.4] shows that $\rho_{D}$ is defined over $\mathbb{Q}$ with respect to $V_{\mathbb{Q}}$ on 
$\mathbb{C} \cdot A_{1} \oplus \mathfrak{c}_{D}^{\prime} \oplus \mathfrak{h}_{D}$. The map $A \mapsto A-d \chi_{D}(A) \cdot A_{1}$ from $\mathbb{C} \cdot A_{1} \oplus \mathfrak{c}_{D}^{\prime} \oplus \mathfrak{h}_{D}$ to $\left(\mathbb{C} \cdot A_{1} \oplus \mathfrak{c}_{D}^{\prime} \oplus \mathfrak{h}_{D}\right) \cap \mathfrak{a}_{D}=\mathfrak{c}_{D}^{\prime} \oplus \mathfrak{h}_{D}$ preserves the property of having eigenvalues in $\mathbb{Q}$. Thus, $\rho_{D}$ is defined over $\mathbb{Q}$ with respect to $V_{\mathbb{Q}}$ also on $\mathfrak{c}_{D}^{\prime} \oplus \mathfrak{h}_{D}$ and hence on $\mathfrak{a}_{D}$. Remark that once $\rho_{D}$ is defined over $\mathbb{Q}$ on $\mathfrak{g}_{D}$, this also holds on the center $\mathbb{C} \cdot A_{1} \oplus \mathfrak{c}_{D}^{\prime}$ of $\mathfrak{g}_{D}$.

\section{$\S 3$. $b$-functions of reductive prehomogeneous determinants}

The (global) b-function of $f \in \mathbb{C}[x]$ is the minimal monic polynomial $B_{f}(s) \in \mathbb{C}[s]$ in a functional equation

$$
B_{f}(s) \cdot f^{s} \in D_{V}[s] \cdot f^{s+1}
$$

where $D_{V}$ denotes the ring of algebraic differential operators on $V$. In general, the existence of the $b$-function has been established by I. N. Bernstein Ber72 in the global case and by J.-E. Björk Bjö79 in the local case. M. Kashiwara [Kas77] proved that the roots of the $b$-function are negative rational numbers.

Let $(G, \rho, V)$ be a reductive prehomogeneous vector space. Then there is a compact Zariski dense subgroup $H \subseteq G$ and one can assume that $H \subseteq U(n)$ for some basis $x$ of $V$. We refer to this basis $x$ and the dual basis $y$ of $V^{*}$ as unitary coordinates. For a relative invariant $f$ of $(G, \rho, V)$ with character $\chi_{f}$, the function $f^{*} \in \operatorname{Sym}(V)$ defined by

$$
f^{*}(y)=\overline{f(\bar{y})}
$$

is then a relative invariant of $\left(G, \rho^{*}, V^{*}\right)$ with character

$$
\chi_{f^{*}}=\chi_{f}^{-1}
$$

by the unitarian trick (see Kim03, §2.3]). In Kim03, Prop. 2.22 and 2.23], this is used to show that $f^{*}$ defines functional equations

$$
\begin{aligned}
f^{*}\left(\partial_{x}\right) \cdot f^{s+1}(x) & =b_{f}(s) \cdot f^{s}(x), \\
f\left(\partial_{y}\right) \cdot\left(f^{*}\right)^{s+1}(y) & =b_{f^{*}}(s) \cdot\left(f^{*}\right)^{s}(y), \\
b_{f}(s) & =b_{f^{*}}(s),
\end{aligned}
$$

as in (3.1) and that

$$
\operatorname{deg} b_{f}(s)=\operatorname{deg} f=n .
$$

From this one deduces that $(G, \rho, V)$ is a regular prehomogeneous vector space and that any relative invariant $f$ defining the discriminant is nondegenerate Kim03, 
Prop. 2.24]. In particular, $\left(G, \rho^{*}, V^{*}\right)$ is again a reductive and hence a regular prehomogeneous vector space. It is shown in Gyo91, Cor. 2.5.10] that

$$
b_{f}(s)=B_{f}(s), \quad b_{f^{*}}(s)=B_{f^{*}}(s) .
$$

Now let $D$ be a (not necessarily reductive) prehomogeneous determinant defined by $f$ as in Definition 2.1. Then we abbreviate

$$
B_{D}(s)=B_{f}(s), \quad b_{D}(s)=b_{f}(s),
$$

where the latter is defined in the presence of the functional equations (3.4).

Definition 3.1. Let $D$ be a prehomogeneous determinant. Consider $G_{D^{*}}=$ $\rho_{D}^{*}\left(G_{D}\right) \subseteq \mathrm{GL}_{V^{*}}$ and $\rho_{D^{*}}=$ id: $G_{D^{*}} \hookrightarrow \mathrm{GL}_{V^{*}}$. Define $D^{*}$ and $f^{*}$ as $D$ and $f$ in Definition 2.1 with $(G, \rho, V)=\left(G_{D^{*}}, \rho_{D^{*}}, V^{*}\right)$. We call $D^{*}$ the dual determinant of $D$. Note that $D^{*}$ is a prehomogeneous determinant exactly if $f^{*} \neq 0$ or $D^{*} \neq V^{*}$.

For $A \in \mathfrak{g}_{D}$, we abbreviate

$$
\delta_{A}^{*}=\left\langle d \rho_{D}^{*}(A) y, \partial_{y}\right\rangle=-y^{t} A \partial_{y}, \quad \delta_{k}=\delta_{A_{k}},
$$

as in 2.5) using (2.4) and 2.8). Note that 2.6) also holds for $f, D, \delta_{A}$ replaced by $f^{*}, D^{*}, \delta_{A}^{*}$.

The following statement is [dGMS09, Prop. 3.7] in the case of linear free divisors.

Proposition 3.2. Together with $D$ also $D^{*}$ is a reductive prehomogeneous determinant (or a reductive linear free divisor) defined by $f^{*}$ in 3.2 .

Proof. In unitary coordinates, we have $\rho_{D}^{*}(g)=\left(\rho_{D}(g)^{-1}\right)^{t}=\overline{\rho_{D}(g)}$ for $g \in H$ and hence $d \rho_{D}^{*}(A)=-d \rho_{D}(A)^{t}=\overline{d \rho_{D}(A)}$ for $A \in \mathfrak{h}$ where $\mathfrak{h}$ denotes the Lie algebra of $H$. As $\mathfrak{h}$ spans $\mathfrak{g}$ over $\mathbb{C}$, it follows that $f^{*}$ of Definition 3.1 coincides with $f^{*}$ of $(3.2)$. This shows that $f^{*} \neq 0$ and that $f^{*}$ is reduced if and only if $f$ is reduced.

If $D$ is a reductive prehomogeneous determinant, the two (equivalent) equalities

$$
\begin{gathered}
\chi_{D^{*}} \circ \rho_{D}^{*}=\chi_{f^{*}}=\chi_{f}^{-1}=\chi_{D}^{-1}, \\
d \chi_{D^{*}} \circ d \rho_{D}^{*}=d \chi_{f^{*}}=-d \chi_{f}=-d \chi_{D}
\end{gathered}
$$

follow from Proposition 3.2 and 3.3 .

The following observation was the starting point for our study of the symmetry of $b$-functions of prehomogeneous determinants in the remainder of this section. 
Theorem 3.3. M. Sato's fundamental theorem for homogeneous vector spaces as formulated in Kim03. Prop. 4.18] holds true for reductive linear free divisors.

Proof. The hypotheses for the proof of this theorem in [Kim03, §4.1] are fulfilled by Theorem 2.5 (c) except for the requirement that $f$ be irreducible. But this latter assumption is used in the proof only to ensure that $f$ and $f^{*}$ can be chosen to be defined over $\mathbb{R}$ (see [Kim03, Prop. 4.1(1), Prop. 4.2]) and that $|\operatorname{det} \rho(g)|=\left|\chi_{f}(g)\right|^{n / d}$ for all $g \in G$ where $d=\operatorname{deg} f$ (see [Kim03, p. 119]). But, by Theorems 2.4 and $2.5 \mathrm{~b}$, these statements hold true for any reductive linear free divisor. Indeed, it follows from Theorem 2.5 a that the Lie algebra representation $d \rho_{D^{*}}: \mathfrak{g}_{D^{*}}=d \rho_{D}^{*}\left(\mathfrak{g}_{D}\right) \hookrightarrow \mathfrak{g l}_{V^{*}}$ is defined over $\mathbb{Q}$ with respect to $V_{\mathbb{Q}}^{*}$. Then the defining equation $f^{*}$ of the dual linear free divisor $D^{*}$ is defined over $\mathbb{Q}$ with respect to $V_{\mathbb{Q}}^{*}$ by Theorem $2.5 \mathrm{~b}$. Note also that the nondegeneracy of $f$ and $f^{*}$ used in [Kim03, Cor. 4.4] follows from [Kim03, Prop. 2.24] even in the reducible case.

A symmetry property of the $b$-function as in Lemma 3.4 below is a corollary of Sato's fundamental theorem (see [Kim03, Prop. 4.19]) for irreducible reductive prehomogeneous vector spaces. For reductive prehomogeneous determinants, we shall give an independent proof which is more elementary in this setting. Our argument also applies to regular linear free divisors (see Section 5).

Lemma 3.4. Let $D$ be a prehomogeneous determinant with $D^{*} \neq V^{*}$ such that (3.4), (3.5), 2.12), and (3.9) hold true. Then its b-function satisfies

$$
b_{D}(s)=(-1)^{n} \cdot b_{D}(-s-2) .
$$

Proof. From (2.6), 2.12, and (3.9) we deduce that

$$
\begin{aligned}
& Q_{A}=\delta_{A}-s \cdot \operatorname{tr}(A)=\delta_{A}-s \cdot \delta_{A}(f) / f \in \operatorname{Ann}_{D_{V}[s]} f^{s}, \\
& Q_{A}^{*}=\delta_{A}^{*}+s \cdot \operatorname{tr}(A)=\delta_{A}^{*}-s \cdot \delta_{A}^{*}\left(f^{*}\right) / f^{*} \in \operatorname{Ann}_{D_{V^{*}}[s]}\left(f^{*}\right)^{s},
\end{aligned}
$$

for $A \in \mathfrak{g}_{D}$ when identifying $A=d \rho_{D}(A)$. So by 3.4 and 3.10 , we have

$$
P=f^{*}\left(\partial_{x}\right) \cdot f(x)-b_{D}(s) \in \operatorname{Ann}_{D_{V}[s]} f^{s} \ni Q_{A_{k}}=Q_{k}
$$

for $k=1, \ldots, n$. Now let $\mathscr{D}_{V}$ denote the sheaf of algebraic differential operators on $V$. Then, at any point $p \in U=V \backslash D$, we have

$$
\operatorname{Ann}_{\mathscr{D}_{V, p}[s]} f^{s}=\left\langle Q_{1}, \ldots, Q_{n}\right\rangle_{\mathscr{D}_{V, p}[s]}
$$

Indeed, as $f \in \mathscr{O}_{V, p}^{*}$, conjugating by $f^{s}$ reduces this statement to $\operatorname{Ann}_{\mathscr{D}_{V, p}[s]} 1=$ $\left\langle\delta_{1}, \ldots, \delta_{n}\right\rangle_{\mathscr{D}_{V, p}[s]}$, which holds true since $\left\langle\delta_{1}, \ldots, \delta_{n}\right\rangle_{\mathscr{O}_{V, p}}=\left\langle\partial_{x_{1}}, \ldots, \partial_{x_{n}}\right\rangle_{\mathscr{O}_{V, p}}$. The 
equality 3.12 gives rise to an exact sequence

$$
0 \rightarrow \mathscr{R} \rightarrow \mathscr{D}_{U}[s]^{n} \stackrel{\left(Q_{1}, \ldots, Q_{n}\right)}{\longrightarrow} \operatorname{Ann}_{\mathscr{D}_{U}[s]} f^{s} \rightarrow 0 .
$$

As $U$ is affine and the sheaf $\mathscr{R}$ is quasicoherent, we have $H^{1}(U, \mathscr{R})=0$ (see Meb89, Prop. 2.5.4] and [Har77, Thm. 3.5]) and this implies together with (3.11) that

$$
f^{m} \cdot P \in\left\langle Q_{1}, \ldots, Q_{n}\right\rangle_{D_{V}[s]}
$$

for some $m \in \mathbb{N}$. Let $\mathscr{F}$ denote the Fourier transform. Then, by 2.5 , [3.8), and 3.10 , we have

$$
\begin{aligned}
\mathscr{F}\left(Q_{A}(s)\right) & =\mathscr{F}\left(x^{t} A^{t} \partial_{x}-s \cdot \operatorname{tr}(A)\right)=-\partial_{y}^{t} A^{t} y-s \cdot \operatorname{tr}(A) \\
& =-y^{t} A \partial_{y}-(s+1) \cdot \operatorname{tr}(A)=\delta_{A}^{*}+(-s-1) \cdot \operatorname{tr}(A) \\
& =Q_{A}^{*}(-s-1) .
\end{aligned}
$$

Combining (3.10), 3.13), and (3.14) yields

$$
\begin{aligned}
\operatorname{Ann}_{D_{V^{*}}[s]}\left(f^{*}\right)^{-s-1} \ni \mathscr{F}\left(f^{m} \cdot P\right) & =\mathscr{F}(f(x))^{m} \cdot \mathscr{F}\left(f^{*}\left(\partial_{x}\right) \cdot f(x)-b_{D}(s)\right) \\
& =(-1)^{n m} f\left(\partial_{y}\right)^{m} \cdot\left((-1)^{n} \cdot f^{*}(y) \cdot f\left(\partial_{y}\right)-b_{D}(s)\right)
\end{aligned}
$$

and hence, using (3.4) and (3.5),

$$
\begin{aligned}
0 & =f\left(\partial_{y}\right)^{m} \cdot\left((-1)^{n} \cdot f^{*}(y) \cdot f\left(\partial_{y}\right)-b_{D}(s)\right) \cdot\left(f^{*}\right)^{-s-1} \\
& =f\left(\partial_{y}\right)^{m} \cdot\left((-1)^{n} \cdot b_{D^{*}}(-s-2)-b_{D}(s)\right) \cdot\left(f^{*}\right)^{-s-1} \\
& =\left((-1)^{n} \cdot b_{D^{*}}(-s-2)-b_{D}(s)\right) \cdot f\left(\partial_{y}\right)^{m} \cdot\left(f^{*}\right)^{-s-1} \\
& =\left((-1)^{n} \cdot b_{D}(-s-2)-b_{D}(s)\right) \cdot b_{D^{*}}(-s-2) \cdots b_{D^{*}}(-s-m-1) \cdot\left(f^{*}\right)^{-s-m-1} .
\end{aligned}
$$

Thus, $(-1)^{n} \cdot b_{D}(-s-2)-b_{D}(s)=0$ as claimed.

We deduce the following from Lemma 3.4 using Theorem 2.4, and from 3.6, (3.7), and [Kas77].

Theorem 3.5. For any reductive prehomogeneous determinant $D \subseteq V$ in dimension $\operatorname{dim} V=n$, the $b$-function $B_{D}(s) \equiv b_{D}(s)$ has degree $n$ and rational negative roots symmetric about -1 . In particular, -1 is the only integer root.

Remark 3.6. In CU04, Prob. 3.2], Castro-Jiménez and Ucha-Enríquez ask whether -1 is the only integer root of the $b$-function for any linear free divisor. Theorem 3.5 gives a positive answer to this question in the case of reductive prehomogeneous determinants. In Theorem 5.5 in Section 5 , we shall also settle the case of certain nonreductive linear free divisors. 


\section{$\S 4$. Examples}

Up to dimension 4 all linear free divisors have been classified in GMNS09, §6.4]. Table 1 lists their $b$-functions computed using Macaulay 2 [M2]. Here $\mathfrak{g}_{2}$ is the non-Abelian Lie algebra of dimension 2 and $\mathfrak{g}_{3}$ is characterized as having twodimensional Abelian derived algebra $\mathfrak{g}_{3}^{\prime}$, on which the adjoint action of a basis vector outside $\mathfrak{g}_{3}^{\prime}$ is semisimple with eigenvalues 1 and 2 (see [Jac79, Ch. I, §4]). Observe that, except for having degree greater than $n$, also the nonreductive divisors in Table 1 satisfy the statement of Theorem 3.5, which supports Conjecture 1.5 .

Table 1: $b$-functions of linear free divisors in dimension $n \leq 4$

\begin{tabular}{ccccc}
\hline$n$ & $f$ & $\mathfrak{g}_{D}$ & reductive? & $\operatorname{Spec} b(s)$ \\
\hline 1 & $x$ & $\mathbb{C}$ & Yes & -1 \\
2 & $x y$ & $\mathbb{C}^{2}$ & Yes & $-1,-1$ \\
3 & $x y z$ & $\mathbb{C}^{3}$ & Yes & $-1,-1,-1$ \\
3 & $\left(y^{2}+x z\right) z$ & $\mathfrak{b}_{2}$ & No & $-\frac{5}{4},-1,-1,-\frac{3}{4}$ \\
4 & $x y z w$ & $\mathbb{C}^{4}$ & Yes & $-1,-1,-1,-1$ \\
4 & $\left(y^{2}+x z\right) z w$ & $\mathbb{C} \oplus \mathfrak{b}_{2}$ & No & $-\frac{5}{4},-1,-1,-1,-\frac{3}{4}$ \\
4 & $(y z+x w) z w$ & $\mathbb{C}^{2} \oplus \mathfrak{g}_{2}$ & No & $-\frac{4}{3},-1,-1,-1,-\frac{2}{3}$ \\
& & & & $-\frac{7}{5},-\frac{4}{3},-\frac{6}{5}$, \\
4 & $x\left(y^{3}-3 x y z+3 x^{2} w\right)$ & $\mathbb{C} \oplus \mathfrak{g}_{3}$ & No & $-1,-1,-1$, \\
& & & & $-\frac{4}{5},-\frac{2}{3},-\frac{3}{5}$ \\
4 & $y^{2} z^{2}-4 x z^{3}-4 y^{3} w+$ & $\mathfrak{g l}_{2}(\mathbb{C})$ & Yes & $-\frac{7}{6},-1,-1,-\frac{5}{6}$ \\
\hline
\end{tabular}

Discriminants of quiver representations are at the origin of linear free divisors and form a rich source of reductive determinants. We shall briefly review the basics of the theory to understand the following three examples and refer to [BM06 for more details.

A quiver $Q=\left(Q_{0}, Q_{1}\right)$ is a directed finite graph with vertex set $Q_{0}$ and edge set $Q_{1} \subseteq Q_{0} \times Q_{0}$. With a dimension vector $\mathbf{d}=\left(d_{i}\right) \in \mathbb{N}^{Q_{0}}$ we associate the representation space

$$
\operatorname{Rep}(Q, \mathbf{d})=\prod_{(i, j) \in Q_{1}} \operatorname{Hom}\left(\mathbb{C}^{d_{i}}, \mathbb{C}^{d_{j}}\right) .
$$

The quiver group

$$
\operatorname{GL}(Q, \mathbf{d})=\prod_{i \in Q_{0}} \mathrm{GL}_{d_{i}}(\mathbb{C})
$$

acts on $\operatorname{Rep}(Q, \mathbf{d})$ through the quiver representation

$$
\begin{gathered}
\rho_{Q, \mathbf{d}}: \operatorname{GL}(Q, \mathbf{d}) \rightarrow \operatorname{GL}(\operatorname{Rep}(Q, \mathbf{d})), \\
\rho_{Q, \mathbf{d}}\left(\left(g_{i}\right)_{i \in Q_{0}}\right)\left(\varphi_{(i, j)}\right)_{(i, j) \in Q_{1}}=\left(g_{j} \circ \varphi_{(i, j)} \circ g_{i}^{-1}\right)_{(i, j) \in Q_{1}} .
\end{gathered}
$$


Note that $Z=\mathbb{C} \cdot\left(I_{d_{i}}\right)_{i \in Q_{0}}$, where $I_{d_{i}} \in \mathrm{GL}_{d_{i}}(\mathbb{C})$ denotes the unit matrix, lies in the kernel of $\rho_{Q, \mathbf{d}}$. The Tits form $q: \mathbb{C}^{Q_{0}} \rightarrow \mathbb{C}$ is defined by

$$
q\left(\left(x_{i}\right)_{i \in Q_{0}}\right)=\sum_{i \in Q_{0}} x_{i}^{2}-\sum_{(i, j) \in Q_{1}} x_{i} x_{j} .
$$

The condition $\operatorname{dim} G=\operatorname{dim} V$ in Definition 2.1 for the discriminant $D$ of $(G, \rho, V)$ $=\left(\mathrm{GL}(Q, \mathbf{d}) / Z, \rho_{Q, \mathbf{d}}, \operatorname{Rep}(Q, \mathbf{d})\right)$ to define a prehomogeneous determinant is equivalent to $q(\mathbf{d})=1$ and reductivity is automatic if $D \neq V$ [GMNS09, §4].

The following example of a linear free divisor is the discriminant of the star quiver studied in GMNS09, Example 5.3]. We use the microlocal calculus developed in SKKŌ81] to determine its $b$-function and then Macaulay 2 [M2 to verify our result.

Example 4.1. Consider the star quiver $Q$ shown in Figure 1 with one sink, three sources, and dimension vector $\mathbf{d}=(2,1,1,1)$ with $q(\mathbf{d})=1$. The quiver group

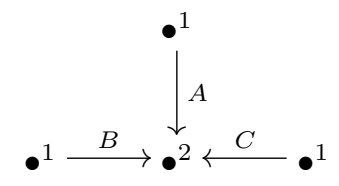

Figure 1. The star quiver in Example 4.1

$G=\mathrm{GL}(Q, \mathbf{d})=\mathrm{GL}_{2}(\mathbb{C}) \times\left(\mathbb{C}^{*}\right)^{3} \subseteq \mathrm{GL}_{2}(\mathbb{C}) \times \mathrm{GL}_{3}(\mathbb{C})$ acts on the 6-dimensional representation space $\operatorname{Rep}(Q, \mathbf{d})=\mathbb{C}^{2 \times 3}=\{(A B C)\}$ of $2 \times 3$-matrices through $\rho=\rho_{Q, \mathbf{d}}$ given by

$$
\rho(g) x=g_{1} \cdot x \cdot g_{2}^{-1}, \quad g=\left(g_{1}, g_{2}\right) .
$$

The infinitesimal vector fields for this group action are given by

$$
\xi_{i, j}=x_{i, 1} \partial_{j, 1}+x_{i, 2} \partial_{j, 2}+x_{i, 3} \partial_{j, 3}, \quad \xi_{k}=x_{1, k} \partial_{1, k}+x_{2, k} \partial_{2, k}
$$

where $x_{i, j}$ are coordinates on $\mathbb{C}^{2 \times 3}$ and $\partial_{i, j}=\partial_{x_{i, j}}$. The discriminant is a reductive linear free divisor $D$ defined by the product of maximal minors

$$
f(x)=\left(x_{1,2} x_{2,3}-x_{2,2} x_{1,3}\right)\left(x_{1,1} x_{2,3}-x_{2,1} x_{1,3}\right)\left(x_{1,1} x_{2,2}-x_{2,1} x_{1,2}\right)
$$

with associated character

$$
\chi(g)=\operatorname{det}\left(g_{1}\right)^{3} \operatorname{det}\left(g_{2}\right)^{-2} .
$$

We first enumerate the different orbits by specifying a generic element $x_{0}$. We denote by $O_{i, j}$ (or $O_{i}$ if there is only one) an orbit of dimension $i$ of a point $x_{0}^{i, j}$ 
(or $x_{0}^{j}$ ), and by $\Lambda_{i, j}$ (or $\Lambda_{i}$ ) its conormal space. In order to calculate the dimension of the orbit of $x_{0}$ it is sufficient to determine the tangent space $T_{x_{0}}=d \rho(\mathfrak{g}) x_{0}$ which is spanned by the evaluation of the vector fields (4.1) at $x_{0}$.

1. Orbits of rank 2:

(1.a) One open orbit:

$$
x_{0}^{6}=\left(\begin{array}{lll}
1 & 0 & 1 \\
0 & 1 & 1
\end{array}\right) .
$$

(1.b) Three orbits of rank 2 and dimension 5 with all columns nonzero:

$$
x_{0}^{5,1}=\left(\begin{array}{lll}
1 & 0 & 0 \\
0 & 1 & 1
\end{array}\right), \quad x_{0}^{5,2}=\left(\begin{array}{lll}
0 & 1 & 0 \\
1 & 0 & 1
\end{array}\right), \quad x_{0}^{5,3}=\left(\begin{array}{lll}
0 & 0 & 1 \\
1 & 1 & 0
\end{array}\right) \text {. }
$$

(1.c) Three orbits of rank 2 and dimension 4 with exactly one zero column:

$$
x_{0}^{4,1}=\left(\begin{array}{lll}
0 & 1 & 0 \\
0 & 0 & 1
\end{array}\right), \quad x_{0}^{4,2}=\left(\begin{array}{lll}
1 & 0 & 0 \\
0 & 0 & 1
\end{array}\right), \quad x_{0}^{4,3}=\left(\begin{array}{lll}
1 & 0 & 0 \\
0 & 1 & 0
\end{array}\right) \text {. }
$$

2. Orbits of rank 1:

(2.a) One orbit of rank 1 and dimension 4:

$$
x_{0}^{4,0}=\left(\begin{array}{lll}
1 & 1 & 1 \\
0 & 0 & 0
\end{array}\right)
$$

2.b) Three orbits of rank 1 and dimension 3:

$$
x_{0}^{3,1}=\left(\begin{array}{lll}
0 & 1 & 1 \\
0 & 0 & 0
\end{array}\right), \quad x_{0}^{3,2}=\left(\begin{array}{lll}
1 & 0 & 1 \\
0 & 0 & 0
\end{array}\right), \quad x_{0}^{3,3}=\left(\begin{array}{lll}
1 & 1 & 0 \\
0 & 0 & 0
\end{array}\right) .
$$

2.c) Three orbits of rank 1 and dimension 2:

$$
x_{0}^{2,1}=\left(\begin{array}{lll}
1 & 0 & 0 \\
0 & 0 & 0
\end{array}\right), \quad x_{0}^{2,2}=\left(\begin{array}{lll}
0 & 1 & 0 \\
0 & 0 & 0
\end{array}\right), \quad x_{0}^{2,3}=\left(\begin{array}{lll}
0 & 0 & 1 \\
0 & 0 & 0
\end{array}\right) .
$$

3. One orbit of rank 0: $x_{0}^{0}=0$.

For each of the above points $x_{0}=x_{0}^{i, j}$, we shall now compute the action $\rho_{x_{0}}$ of the isotropy group $G_{x_{0}}$ on the normal space $V_{x_{0}}=V / T_{x_{0}}$ to the tangent space $T_{x_{0}}$ to the orbit of $x_{0}$. We omit the trivial cases of the open orbit and of the zero orbit and describe $V_{x_{0}}$ by identifying it with a representative subspace in $V$. 
1. $x_{0}=x_{0}^{5,1}$ :

$$
\begin{gathered}
G_{x_{0}}=\left\{g=\left(\begin{array}{ll}
a & 0 \\
0 & 1
\end{array}\right) \times(a, 1,1)\right\}, \quad T_{x_{0}}=\left\langle\partial_{1,1}, \partial_{2,1}, \partial_{2,2}, \partial_{2,3}, \partial_{1,2}+\partial_{1,3}\right\rangle, \\
V_{x_{0}}=\left\{x=\left(\begin{array}{lll}
0 & 0 & u \\
0 & 0 & 0
\end{array}\right)\right\}, \quad \rho_{x_{0}}(g) x=a \cdot x .
\end{gathered}
$$

2. $x_{0}=x_{0}^{4,3}$ :

$$
\begin{gathered}
G_{x_{0}}=\left\{g=\left(\begin{array}{cc}
1 & 0 \\
0 & a
\end{array}\right) \times(1, a, b)\right\}, \quad T_{x_{0}}=\left\langle\partial_{1,1}, \partial_{1,2}, \partial_{2,1}, \partial_{2,2}\right\rangle, \\
V_{x_{0}}=\left\{x=\left(\begin{array}{lll}
0 & 0 & u \\
0 & 0 & v
\end{array}\right)\right\}, \quad \rho_{x_{0}}(g) x=\frac{1}{b}\left(\begin{array}{ccc}
0 & 0 & u \\
0 & 0 & a v
\end{array}\right) .
\end{gathered}
$$

3. $x_{0}=x_{0}^{4,0}$ :

$$
\begin{gathered}
G_{x_{0}}=\left\{g=\left(\begin{array}{ll}
1 & a \\
0 & b
\end{array}\right)\right\}, \quad T_{x_{0}}=\left\langle\partial_{1,1}, \partial_{1,2}, \partial_{1,3}, \partial_{2,1}+\partial_{2,2}+\partial_{2,3}\right\rangle, \\
V_{x_{0}}=\left\{x=\left(\begin{array}{ccc}
0 & 0 & 0 \\
0 & u & v
\end{array}\right)\right\}, \quad \rho_{x_{0}}(g) x=b \cdot x .
\end{gathered}
$$

This is the only non-prehomogeneous normal action.

4. $x_{0}=x_{0}^{3,3}$ :

$$
\begin{array}{ccc}
G_{x_{0}}=\left\{g=\left(\begin{array}{ll}
1 & a \\
0 & b
\end{array}\right) \times(1,1, c)\right\}, & T_{x_{0}}=\left\langle\partial_{1,1}, \partial_{1,2}, \partial_{2,1}+\partial_{2,2}\right\rangle, \\
V_{x_{0}}=\left\{x=\left(\begin{array}{lll}
0 & 0 & u \\
0 & v & w
\end{array}\right)\right\}, & \rho_{x_{0}}(g) x=\left(\begin{array}{cc}
0 & (u+a w) / c \\
b v & b w / c
\end{array}\right) .
\end{array}
$$

This is a prehomogeneous action with nonreduced discriminant $\left\{v w^{2}=0\right\}$.

5. $x_{0}=O_{2,1}$ :

$$
\begin{gathered}
G_{x_{0}}=\left\{g=\left(\begin{array}{ll}
1 & a \\
0 & b
\end{array}\right) \times(1, c, d)\right\}, \quad T_{x_{0}}=\left\langle\partial_{11}, \partial_{21}\right\rangle \\
V_{x_{0}}=\left\{x=\left(\begin{array}{lll}
0 & r & s \\
0 & t & u
\end{array}\right)\right\}, \quad \rho_{x_{0}}(g) x=\left(\begin{array}{ccc}
0 & (r+a t) / c & (s+a u) / d \\
0 & b t / c & b u / d
\end{array}\right)
\end{gathered}
$$

This is a prehomogeneous action with reduced discriminant $\{t u(u r-s t)=0\}$.

The holonomy diagram in Figure 2 shows the incidence relations between the good orbit conormals subject to intersections in codimension one. Recall that $\Lambda_{4,0}$ is not good as it is not prehomogeneous. We shall apply the method of [SKKŌ81] to the 


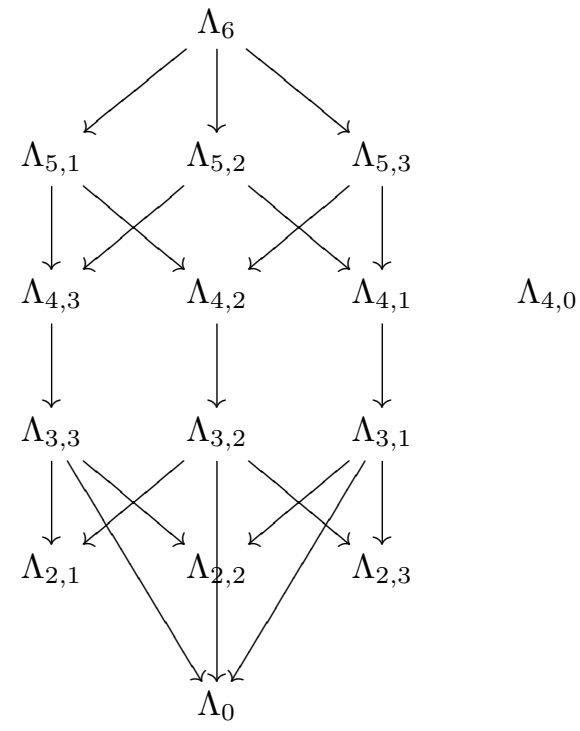

Figure 2. Holonomy diagram of Example 4.1

chain

$$
\Lambda_{6} \rightarrow \Lambda_{5,1} \rightarrow \Lambda_{4,3} \rightarrow \Lambda_{3,3} \rightarrow \Lambda_{0}
$$

An elementary local calculation shows that all the intersections in this chain are

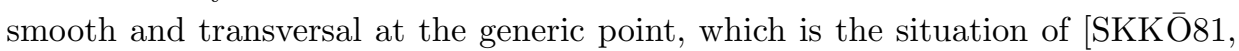
Cor. 7.6]. Therefore it remains to calculate $\operatorname{ord}_{\Lambda} f^{s}$ for all $\Lambda$ in the chain (4.3) using the following formula from [SKKŌ81, Prop. 4.14]:

$$
\operatorname{ord}_{\Lambda} f^{s}=-m_{\Lambda} s-\frac{\mu_{\Lambda}}{2}=d \chi\left(A_{0}\right)-\operatorname{tr}_{V_{x_{0}}^{*}} d \rho_{x_{0}}^{*}\left(A_{0}\right)+\frac{1}{2} \operatorname{dim} V_{x_{0}}^{*}
$$

Here $A_{0}=\left(A_{0}^{1}, A_{0}^{2}\right) \in \mathfrak{g}_{x_{0}}$ and $\left(x_{0}, y_{0}\right) \in \Lambda$ is generic such that $d \rho_{x_{0}}\left(A_{0}\right) y_{0}=y_{0}$. By 4.2 , we have

$$
d \chi\left(A_{0}\right)=3 \cdot \operatorname{tr}\left(A_{0}^{1}\right)-2 \cdot \operatorname{tr}\left(A_{0}^{2}\right), \quad \operatorname{tr}_{V_{x_{0}}^{*}} d \rho_{x_{0}}^{*}\left(A_{0}\right)=-\operatorname{tr}_{V_{x_{0}}} d \rho_{x_{0}}\left(A_{0}\right),
$$

which can be determined from the preceding computations. Note that the contragredient action $\rho^{*}$ on the dual space $\left(\mathbb{C}^{2 \times 3}\right)^{*}$ identified with $\mathbb{C}^{3 \times 2}$ by the trace pairing is given by

$$
d \rho^{*}(g) \xi=g_{2} \xi g_{1}^{-1}, \quad g=\left(g_{1}, g_{2}\right)
$$


One computes

$$
\begin{gathered}
\operatorname{ord}_{\Lambda_{6}} f^{s}=0, \quad \operatorname{ord}_{\Lambda_{5,1}} f^{s}=-s-\frac{1}{2}, \quad \operatorname{ord}_{\Lambda_{4,3}} f^{s}=-2 s-1, \\
\operatorname{ord}_{\Lambda_{3,3}} f^{s}=-5 s-\frac{5}{2}, \quad \operatorname{ord}_{\Lambda_{0}} f^{s}=-6 s-3 .
\end{gathered}
$$

Using the formula in SKKŌ81, Cor. 7.6] this yields

$$
\begin{gathered}
\frac{b_{\Lambda_{5,1}}}{b_{\Lambda_{6}}}=s+1, \quad \frac{b_{\Lambda_{4,3}}}{b_{\Lambda_{5,1}}}=-s-\frac{1}{2}+2 s+1+\frac{1}{2}=s+1 \\
\frac{b_{\Lambda_{3,3}}}{b_{\Lambda_{4,3}}}=(3 s+2)(3 s+3)(3 s+4), \quad \frac{b_{\Lambda_{0}}}{b_{\Lambda_{3,3}}}=-5 s-\frac{5}{2}+6 s+3+\frac{1}{2}=s+1 .
\end{gathered}
$$

From this we conclude that

$$
B_{D}(s)=b_{\Lambda_{0}}(s)=\left(s+\frac{2}{3}\right)(s+1)^{4}\left(s+\frac{4}{3}\right)
$$

is the $b$-function of $D$, which is also confirmed by Macaulay 2 M2].

The next two examples are reductive nonreduced prehomogeneous determinants arising from quiver representations.

Example 4.2. We modify the star quiver of Example 4.1 by adding an additional source. Consider the resulting star quiver $\widetilde{D}_{3}$ in Figure 3 and the dimension vector

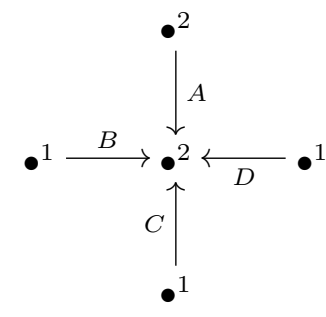

Figure 3. The star quiver in Example 4.2

$\mathbf{d}=(2,2,1,1,1)$ with $q(\mathbf{d})=1$. The quiver group $\mathrm{GL}(Q, \mathbf{d})=\mathrm{GL}_{2}(\mathbb{C}) \times \mathrm{GL}_{2}(\mathbb{C}) \times$ $\left(\mathbb{C}^{*}\right)^{3}$ acts on a 10-dimensional representation space $\operatorname{Rep}(Q, \mathbf{d})=\mathbb{C}^{2 \times 2} \times \mathbb{C}^{3 \times 2}=$ $\{(A,(B C D))\}$ and the orbit of $\left(\left(\begin{array}{ll}1 & 0 \\ 0 & 1\end{array}\right),\left(\begin{array}{lll}1 & 0 & 1 \\ 0 & 1 & 1\end{array}\right)\right)$ is open. The nonreduced discriminant $D$ defined by the function

$$
\begin{aligned}
f(x)= & \left(x_{1,1} x_{2,2}-x_{1,2} x_{2,1}\right)^{2} \cdot\left(x_{1,3} x_{2,4}-x_{1,4} x_{2,3}\right) \cdot\left(x_{1,3} x_{2,5}-x_{1,5} x_{2,3}\right) \\
& \cdot\left(x_{1,4} x_{2,5}-x_{1,5} x_{2,4}\right)
\end{aligned}
$$


is a reductive prehomogeneous determinant. It is a union $D=D_{1} \cup D_{2}$ where $D_{1}$ and $D_{2}$ are defined by the functions

$$
\begin{aligned}
& f_{1}(x)=\left(x_{1,1} x_{2,2}-x_{1,2} x_{2,1}\right)^{2}, \\
& f_{2}(x)=\left(x_{1,3} x_{2,4}-x_{1,4} x_{2,3}\right) \cdot\left(x_{1,3} x_{2,5}-x_{1,5} x_{2,3}\right) \cdot\left(x_{1,4} x_{2,5}-x_{1,5} x_{2,4}\right) .
\end{aligned}
$$

Since $D_{1}$ is the square of a quadratic form and $D_{2}$ is the reductive linear free divisor from Example 4.1, we have

$$
\begin{aligned}
& B_{D_{1}}(s)=(2 s+1)(s+1)^{2}(2 s+3), \\
& B_{D_{2}}(s)=\left(s+\frac{2}{3}\right)(s+1)^{4}\left(s+\frac{4}{3}\right) .
\end{aligned}
$$

As the functions in 4.5 depend on separate sets of variables, $B_{D}(s)$ divides $B_{D_{1}}(s) \cdot B_{D_{2}}(s)$ and hence

$$
B_{D}=B_{D_{1}} \cdot B_{D_{2}}=\left(s+\frac{1}{2}\right)\left(s+\frac{2}{3}\right)(s+1)^{6}\left(s+\frac{4}{3}\right)\left(s+\frac{3}{2}\right)
$$

as $\operatorname{deg} B_{D}=10=\operatorname{deg} B_{D_{1}}+\operatorname{deg} B_{D_{2}}$ by Theorem 3.5. For the reduced discriminant $D_{\text {red }}$, we loose the symmetry property. Indeed, one computes

$$
B_{D_{\text {red }}}(s)=\left(s+\frac{2}{3}\right)(s+1)^{5}\left(s+\frac{4}{3}\right)(s+2)
$$

using the same degree argument.

Example 4.3. Consider the quiver representation associated with the quiver $\widetilde{A}_{n}$ in Figure 4 and the dimension vector $\mathbf{d}=(2,1, \ldots, 1)$ with $q(\mathbf{d})=1$. The repre-

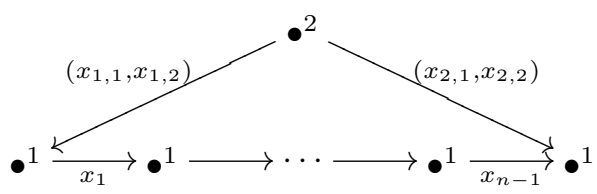

Figure 4. The quiver in Example 4.3

sentation space is $(n+3)$-dimensional and the orbit of

$$
\left(\left(\begin{array}{ll}
x_{1,1} & x_{1,2} \\
x_{2,1} & x_{2,2}
\end{array}\right),\left(x_{1}, \ldots, x_{n-1}\right)\right)=\left(\left(\begin{array}{ll}
1 & 0 \\
0 & 1
\end{array}\right),(1, \ldots, 1)\right)
$$

is open. The nonreduced discriminant $D$ defined by the function

$$
f(x)=\operatorname{det}\left(\begin{array}{ll}
x_{1,1} & x_{1,2} \\
x_{2,1} & x_{2,2}
\end{array}\right)^{2} \cdot x_{1} \cdots x_{n-1}=\left(x_{1,1} x_{2,2}-x_{1,2} x_{2,1}\right)^{2} \cdot x_{1} \cdots x_{n-1}
$$


is a reductive prehomogeneous determinant. As in Example 4.2, one computes

$$
\begin{aligned}
B_{D}(s) & =\left(s+\frac{1}{2}\right)(s+1)^{n+1}\left(s+\frac{3}{2}\right), \\
B_{D_{\text {red }}}(s) & =(s+1)^{n}(s+2),
\end{aligned}
$$

and the reduced discriminant does not have the symmetry property.

\section{$\S 5$. $b$-functions of special linear free divisors}

The symmetry of the $b$-functions of nonreductive prehomogeneous determinants in Section 4 motivates the attempt to weaken the hypotheses of Theorem 3.5 In the case of linear free divisors, this turns out to be possible.

Consider a linear free divisor $D$ defined by $f$ as in Definition 2.1. In GMNS09, Thm. 6.1], we proved that $\mathfrak{a}_{D}$ has a basis $A_{2}, \ldots, A_{n}=S_{2}, \ldots, S_{s}, N_{1}, \ldots, N_{n-r}$ as in the proof of Theorem 2.4 the $d \rho_{D}\left(S_{i}\right)$ are semisimple and commute, the $d \rho_{D}\left(N_{j}\right)$ are nilpotent, and the $N_{J}$ are homogeneous with respect to the $S_{i}$ with rational weights. Then, as in 2.13, we have

$$
d \chi_{f}\left(A_{i}\right)=\operatorname{tr} \circ d \rho_{D}\left(A_{i}\right)+\sum_{j=1}^{n}\left[A_{i}, A_{j}\right] / A_{j}, \quad i=1, \ldots, s .
$$

Assume now that $D^{*} \neq V^{*}$. Then the analog of (5.1) for $D^{*}$ reads

$$
d \chi_{f^{*}}\left(A_{i}\right)=\operatorname{tr} \circ d \rho_{D}^{*}\left(A_{i}\right)+\sum_{j=1}^{n}\left[A_{i}, A_{j}\right] / A_{j}, \quad i=1, \ldots, s .
$$

As $\operatorname{tr} \circ d \rho_{D}^{*}\left(A_{i}\right)=-\operatorname{tr} \circ d \rho_{D}\left(A_{i}\right)$, we obtain the equalities

$$
d \chi_{f}\left(A_{i}\right)-d \chi_{f^{*}}\left(A_{i}\right)=2 \operatorname{tr} \circ d \rho_{D}\left(A_{i}\right), \quad i=1, \ldots, n,
$$

as the difference of 5.1) and 5.2 for $i=1, \ldots, s$. Note that both sides of 5.3 . are zero for $i=s+1, \ldots, n$. From (5.3), we deduce the following formulas.

Lemma 5.1. For a linear free divisor $D$ with $D^{*} \neq V^{*}$,

$$
\chi_{f} \cdot \chi_{f^{*}}^{-1}=\operatorname{det}^{2} \circ \rho_{D}, \quad d \chi_{f}-d \chi_{f^{*}}=2 \operatorname{tr} \circ d \rho_{D} .
$$

As an immediate consequence of Lemma 5.1, (2.12) and (3.9) are equivalent.

Proposition 5.2. For a linear free divisor $D$ with $D^{*} \neq V^{*}$, we have $\chi_{D}=$ $\operatorname{det} \circ \rho_{D}$ if and only if $\chi_{f^{*}}=\chi_{f}^{-1}$. 
A relation of $(3.4),(3.5)$, and $(3.9)$ can be established for general prehomogeneous determinants.

Proposition 5.3. Let $D$ be a prehomogeneous determinant with $D^{*} \neq V^{*}$. Then (3.4) is equivalent to 3.9 and implies 3.5.

Proof. The classical proof essentially works in our setting:

The existence of $b_{f}(s)$ and $b_{f^{*}}(s)$ satisfying (3.4) follows from 3.9 by the character argument of [Kim03, Prop. 2.22], which also gives the converse implication.

In the reductive case,

$$
f(x)=\sum_{|\alpha|=n} f_{\alpha} \cdot x^{\alpha}, \quad f^{*}(y)=\sum_{|\alpha|=n} f_{\alpha}^{*} \cdot x^{\alpha}
$$

have complex conjugate coefficients $f_{\alpha}=\overline{f_{\alpha}^{*}}$ in unitary coordinates. This is not necessary to show that

$$
b_{f}(0)=f^{*}\left(\partial_{x}\right) \cdot f(x)=\sum_{|\alpha|=n} f_{\alpha} \cdot f_{\alpha}^{*} \cdot \alpha !=f\left(\partial_{y}\right) \cdot f^{*}(y)=b_{f^{*}}(0)
$$

and similarly

$b_{f}(0) \cdots b_{f}(m-1)=f^{*}\left(\partial_{x}\right)^{m} \cdot f(x)^{m}=f\left(\partial_{y}\right)^{m} \cdot f^{*}(y)^{m}=b_{f^{*}}(0) \cdots b_{f^{*}}(m-1)$

for any $m \in \mathbb{N}$. The equality in (3.5) follows as in Kim03, Prop. 2.23].

In dGMS09, Def. 2.1], a linear free divisor is called special if 2.12 holds true. Using this terminology, we deduce from Lemma 3.4 and Propositions 5.2 and 5.3 the following result analogous to Theorem 3.5

Theorem 5.4. Let $D \subseteq V$ be a special linear free divisor in dimension $\operatorname{dim} V=n$ with $D^{*} \neq V^{*}$. Then the $b$-function $b_{D}(s)$ has degree at most $n$ and roots symmetric about -1 .

Under the stronger assumption of regularity we can prove more.

Theorem 5.5. Let $D \subseteq V$ be a regular special linear free divisor in dimension $\operatorname{dim} V=n$. Then the $b$-function $B_{D}(s) \equiv b_{D}(s)$ has degree at most $n$ and negative rational roots symmetric about -1 . In particular, -1 is the only integer root.

Proof. Note that the hypothesis $D^{*} \neq V^{*}$ in Theorem 5.4 becomes redundant for regular $D$ by Kim03, Thm. 2.16].

By SKKŌ81, Prop. 4.6], regularity of $D$ is equivalent to $V^{*}=T_{0}^{*} V$ being a good holonomic variety. Under this latter hypothesis the arguments in Gyo91, Lem. 2.5.7] still yield (3.7). 
Note that in Theorem 5.5 regularity of $f$ is equivalent to $\operatorname{deg} b_{D}(s)=n$. Indeed, the degree $n$ coefficient of $b_{f}(s)$ is given by $f^{*}(\operatorname{grad} \log f) \cdot f$ by $\underline{\operatorname{Kim03}}$, Prop. 2.13(2)] and $\operatorname{grad} \log f: V \rightarrow V^{*}$ is a $G$-equivariant map by Kim03, Prop. 2.13(1)].

\section{$\S 6$. Euler homogeneity and normal representations}

The condition of strong Euler homogeneity arose in the context of the logarithmic comparison theorem in CMNCJ02, Conj. 1.4] (see also CNM96 and GS06) and has been studied in GMNS09, §7] in the case of linear free divisors. It is conceivable that all linear free divisors are strongly Euler homogeneous, but there is neither a proof nor a counter-example.

Definition 6.1. Let $D \subseteq V$ be a reduced hypersurface. A (local) vector field $\epsilon \in \operatorname{Der}(-\log D)$ is called an Euler vector field for $D$ if $\epsilon(f)=f$ for some (local) defining equation $f$ of $D$. It is called an Euler vector field at $p \in D$ if in addition $\epsilon(p)=0$. The hypersurface $D$ is called strongly Euler homogeneous at $p \in D$ if there is an Euler vector field at $p$ defined locally at $p$. By strong Euler homogeneity of $D$ we mean that this property holds for all $p \in D$.

The following statement is implicit in the proof of [GMNS09, Lem. 7.5].

Lemma 6.2. If $D$ is (locally) defined by $f$ and Euler homogeneous with Euler vector field $\epsilon$ then $D$ is strongly Euler homogeneous if $\epsilon(p) \in \operatorname{Der}(-\log f)(p)$ for all $p \in D$.

Assume now that $D$ is a linear free divisor. Then the preceding observation can be formulated as follows.

Lemma 6.3. Strong Euler homogeneity of a linear free divisor $D$ means that the $G_{D}$-orbits and $A_{D}$-orbits in $D$ coincide.

We shall now give a sufficient condition for strong Euler homogeneity of $D$ at $x_{0} \in D$. Let $G_{D, x_{0}}$ be the stabilizer of $x_{0}$ in $G_{D}$ with Lie algebra $\mathfrak{g}_{D, x_{0}}$ and consider the representation $\rho_{D, x_{0}}: G_{D, x_{0}} \rightarrow V_{x_{0}}$ induced by $\rho_{D}$ on the normal space $V_{x_{0}}=V / d \rho_{D}\left(\mathfrak{g}_{D}\right) x_{0}$ to the tangent space of the $G_{D}$-orbit of $x_{0}$ at $x_{0}$.

The localization $f_{x_{0}} \in \operatorname{Sym}\left(V^{*}\right)$ of $f$ at $x_{0}$ [SKKŌ81, Def. 6.7] is the homogeneous nonzero polynomial defined by

$$
f\left(x_{0}+\varepsilon x^{\prime}\right) \equiv \varepsilon^{k} \cdot f_{x_{0}}\left(x^{\prime}\right) \bmod \left\langle\varepsilon^{k+1}\right\rangle .
$$

By SKKŌ81, Def. 6.8], $f_{x_{0}}\left(x^{\prime}\right)$ induces a relative invariant $f_{x_{0}}(y)$ of the representation $\left(G_{D, x_{0}}, \rho_{D, x_{0}}, V_{x_{0}}\right)$ with character $\chi_{f_{x_{0}}}=\chi_{D}$ where $y=y_{1}, \ldots, y_{k}$ are 
coordinates on $V_{x_{0}}$. Note however that the latter might not be a prehomogeneous vector space.

In the context of linear free divisors, Definition 2.1 applied to $(G, \rho, V)=$ $\left(G_{D, x_{0}}, \rho_{D, x_{0}}, V_{x_{0}}\right)$, as in Definition 3.1. gives another natural candidate $D_{x_{0}}$ for such an invariant.

Definition 6.4. Assume that $A_{1}, \ldots, A_{k}$ form a basis of $\mathfrak{g}_{D, x_{0}}$ and define $D_{x_{0}}$ $\subseteq V_{x_{0}}$ by

$$
f_{x_{0}}^{\prime}(y)=\operatorname{det}\left(d \rho_{D, x_{0}}\left(A_{1}\right) y, \ldots, d \rho_{D, x_{0}}\left(A_{k}\right) y\right) \in \operatorname{Sym}\left(V_{x_{0}}^{*}\right) .
$$

Then $f_{x_{0}}^{\prime} \neq 0$ or $D_{x_{0}} \neq V_{x_{0}}$ is equivalent to $\left(G_{D, x_{0}}, \rho_{D, x_{0}}, V_{x_{0}}\right)$ being a prehomogeneous vector space.

Lemma 6.5. Let $D$ be a linear free divisor and assume that $x_{0} \in D$ and $D_{x_{0}}$ $\neq V_{x_{0}}$. Then $f_{x_{0}}^{\prime}(y)=f_{x_{0}}(y)$.

Proof. Note that $A_{k+1} x_{0}, \ldots, A_{n} x_{0}$ form a basis of the tangent space $d \rho_{D}\left(\mathfrak{g}_{D}\right) x_{0}$ to the $G_{D}$-orbit of $x_{0}$ at $x_{0}$. We may assume that $d \rho_{D}\left(\mathfrak{g}_{D}\right) x_{0}=0 \times \mathbb{C}^{n-k}$ and then that

$$
\left(A_{k+1} x_{0}, \ldots, A_{n} x_{0}\right)=\left(\begin{array}{ccc}
0 & \ldots & 0 \\
\vdots & & \vdots \\
0 & \ldots & 0 \\
1 & \ldots & 0 \\
\vdots & \ddots & \vdots \\
0 & \ldots & 1
\end{array}\right) .
$$

We may then choose $y=x_{1}^{\prime}, \ldots, x_{k}^{\prime}$. Using (2.1) and (6.1), we compute

$$
\begin{aligned}
f\left(x_{0}+\varepsilon x^{\prime}\right) & =\operatorname{det}\left(A_{1}\left(x_{0}+\varepsilon x^{\prime}\right), \ldots, A_{n}\left(x_{0}+\varepsilon x^{\prime}\right)\right) \\
& \equiv \varepsilon^{k} \cdot \operatorname{det}\left(A_{1} x^{\prime}, \ldots, A_{k} x^{\prime}, A_{k+1} x_{0}, \ldots, A_{n} x_{0}\right) \bmod \left\langle\varepsilon^{k+1}\right\rangle .
\end{aligned}
$$

If we denote by $A_{i}^{\prime}$ the matrix consisting of the upper $k$ rows of $A_{i}, \sqrt{6.1}$ implies that

$$
\begin{aligned}
& \operatorname{det}\left(A_{1} x^{\prime}, \ldots, A_{k} x^{\prime}, A_{k+1} x_{0}, \ldots, A_{n} x_{0}\right)=\operatorname{det}\left(A_{1}^{\prime} x^{\prime}, \ldots, A_{k}^{\prime} x^{\prime}\right) \\
& \quad \equiv \operatorname{det}\left(d \rho_{D, x_{0}}\left(A_{1}\right) y, \ldots, d \rho_{D, x_{0}}\left(A_{k}\right) y\right)=f_{x_{0}}^{\prime}(y) \bmod \left\langle x_{k+1}, \ldots, x_{n}\right\rangle .
\end{aligned}
$$

By hypothesis, $f_{x_{0}}^{\prime} \neq 0$ and the claim follows from 6.2 and 6.3 .

Proposition 6.6. Let $D$ be a linear free divisor and assume that $x_{0} \in D$ and $D_{x_{0}} \neq V_{x_{0}}$. Then $D$ is strongly Euler homogeneous at $x_{0}$. 
Proof. By assumption $\left(G_{D, x_{0}}, \rho_{D, x_{0}}, V_{x_{0}}\right)$ is a prehomogeneous vector space and $f_{x_{0}}(y)$ is a nonzero relative invariant with character $\chi_{D}$. Thus, there must be an infinitesimal vector field as in (2.5) that is not tangent to the level sets of $f_{x_{0}}(y)$. By 2.6, this means that $\chi_{D}(B)=1$ for some $B \in \mathfrak{g}_{x_{0}}$. Using (2.2) and 2.4 we conclude that $B=A_{1}-A$ with $A \in \mathfrak{a}_{D}$ and hence $\rho_{D}\left(A_{1}\right) x_{0}=\rho_{D}(A) x_{0}$ by definition of $\mathfrak{g}_{x_{0}}$. Then the claim follows from Lemma 6.2 .

\section{$\S 7$. Koszul freeness and Euler homogeneity}

Koszul freeness is a natural finiteness condition on logarithmic vector fields and is also closely related to the logarithmic comparison theorem (see Tor04 and CN05). In geometric terms, Koszul freeness of a free divisor $D \subseteq V$ can be defined as the local finiteness of the logarithmic stratification by maximal integral submanifolds along logarithmic vector fields as defined in [Sai80, §3]. As shown in GMNS09, Thm. 7.4], this condition can be formulated algebraically as the symbols of a basis of $\operatorname{Der}(-\log D) \subseteq \mathscr{D}_{V}$ forming a regular sequence in $\operatorname{gr}_{F} \mathscr{D}_{V}=\mathscr{O}_{V} \otimes \operatorname{Sym}(V)$ where $F$ denotes the order filtration on $\mathscr{D}_{V}$. Without the technical details, this equivalence can be understood as follows: At each point $p \in V$, $\operatorname{Der}(-\log D)(p)$ spans the tangent space of the logarithmic stratum through $p$. Thus, the logarithmic variety in $T^{*} V$ defined by the symbols of local bases of $\operatorname{Der}(-\log D)$ is the union of conormals to the logarithmic strata (see [Sai80, (3.16)]). It is of (co)dimension $n$ exactly where the symbols of $n$ local basis elements of $\operatorname{Der}(-\log D)$ form a regular sequence in the Cohen-Macaulay coordinate ring $\mathscr{O}_{V} \otimes \operatorname{Sym}(V)$ of $T^{*} V$ (see [CN02, 1.8]).

It is tempting to introduce a stronger version of Koszul freeness by imposing local finiteness of the logarithmic stratification of $\operatorname{Der}(-\log f)$ on $D$. But unless $D$ is strongly Euler homogeneous, which reduces this condition to ordinary Koszul freeness by Lemma 6.2 it is not clear that the condition in question depends only on $D$.

For a linear free divisor $D$, the logarithmic stratification is the stratification by orbits of $G_{D}$ consisting of smooth locally closed algebraic varieties (see Hum75, Prop. 8.3]). Using the action of $\mathbb{C}^{*} \subseteq G_{D}$, one can see that local finiteness of this stratification is equivalent to finiteness. Moreover, there is a natural choice of defining equation which we use to introduce the desired strong version of Koszul freeness for this class of divisors.

Definition 7.1. We call a linear free divisor $D$ Koszul free if the stratification of $D$ by orbits of $G_{D}$ is finite, and strongly Koszul free if the same holds for the stratification by orbits of $A_{D}$. 
Note that the $G_{D}$-orbit $V \backslash D$ is a union of infinitely many $A_{D}$-orbits by reasons of dimension. The algebraic definition of Koszul freeness above translates to strong Koszul freeness as follows.

Proposition 7.2. A linear free divisor $D$ is Koszul free if and only if the symbols of a basis of $\mathfrak{g}_{D}$ form a regular sequence in $\operatorname{gr}_{F} D_{V}$. It is strongly Koszul free if and only if the symbols of a basis of $\mathfrak{a}_{D}$ and $f$ form a regular sequence in $\operatorname{gr}_{F} D_{V}$.

Proof. Let $\delta_{2}, \ldots, \delta_{n}$ be a basis of $\mathfrak{a}_{D}$ and denote by $\sigma_{F}: D_{V} \rightarrow \operatorname{gr}_{F} D_{V}$ the symbol map for the order filtration $F$ on $D_{V} \cdot \operatorname{As}_{\operatorname{gr}_{F}} D_{V}=\operatorname{Sym}\left(V^{*} \oplus V\right)$ is a polynomial ring hence Cohen-Macaulay, we have to show that the variety $\operatorname{Var}\left(f, \sigma_{F}\left(\delta_{2}\right), \ldots, \sigma_{F}\left(\delta_{n}\right)\right)$ has (co)dimension $n$ exactly if the stratification of $D$ by orbits of $A_{D}$ is finite. As $\mathfrak{a}_{D}$ spans the tangent spaces of the orbits, $\operatorname{Var}\left(\sigma_{F}\left(\delta_{2}\right), \ldots, \sigma_{F}\left(\delta_{n}\right)\right)$ is the union of conormals to the orbits and $\operatorname{Var}(f)=$ $\left.T^{*} V\right|_{D}$. The second claim follows and the proof of the first one is similar.

Remark 7.3. The following generalization of strong Koszul freeness to arbitrary free divisors has been suggested to us by L. Narváez Macarro: Let $D \subseteq X$ be a free divisor in a complex manifold. Then we call $D$ strongly Koszul free if, locally at any point $p \in D$, and for some (and hence any) reduced local defining equation $f$ of $D$ at $p$ and for some (and hence any) local basis $\delta_{1}, \ldots, \delta_{n}$ of $\operatorname{Der}(-\log D)$, the symbols of $f$ and $\delta_{i}-\delta_{i}(f) / f \cdot s, i=1, \ldots, n$, with respect to the (total) order filtration $F$ form a regular sequence in $\operatorname{gr}_{F} \mathscr{D}_{X, p}[s]=\operatorname{Sym}_{\mathscr{O}_{X, p}} F^{1} \mathscr{D}_{X, p}$. By [NM08, Prop. 2.2.16] and Proposition 7.2 this reduces to Definition 7.1 in the special case. Using ideas of A. Simis and T. Torrelli [CN09, Prop. 1.27], Narváez Macarro can prove that strong Koszul freeness is equivalent to being free with Jacobian ideal of linear type.

Let us now relate the properties of strong Euler homogeneity, Koszul freeness, and strong Koszul freeness for linear free divisors.

Proposition 7.4. The following implications hold for any linear free divisor D.

(a) Strong Koszul freeness implies Koszul freeness.

(b) Strong Koszul freeness implies strong Euler homogeneity.

(c) For Koszul free D, strong Euler homogeneity and strong Koszul freeness are equivalent.

Proof. (a) is clear by Definition 7.1 and (c) follows from (b) and Lemma 6.3 .

For $0 \leq k \leq n-1$, let $T_{k}=\left\{p \in D \mid \operatorname{rk}_{D}(p) \leq k\right\}$ (see [GMNS09, §7] and

Sai80, Def. 3.12]). Then $T_{k}$ is a homogeneous algebraic variety defined by $f$ and the $(k+1) \times(k+1)$-minors of the matrix $\left(\delta_{i}\right)_{i=2, \ldots, n}$, and equals the union of the 
$A_{D}$-orbits of dimension at most $k$. As the Euler vector field $\epsilon$ is the infinitesimal generator of the $\mathbb{C}^{*}$-action that defines homogeneity, $\epsilon$ is tangent to $T_{k}$ and to all $G_{D}$-orbits. Therefore, $T_{k}$ is also a union of $G_{D^{-}}$orbits. So if $D$ is strongly Koszul free, $T_{k} \backslash T_{k-1}$ is the finite union of all $k$-dimensional $A_{D}$-orbits each of which is also a $G_{D}$-orbit. This proves (b).

Finally, we are ready to prove our main application to the LCT problem.

Proof of Theorem 1.6. Let $D$ be a reductive linear Koszul free divisor defined by $f$. Because of the $\mathbb{C}^{*}$-action, the (global) $b$-function of $D$ coincides with the local $b$ function of the germ $f_{0}$ of $f$ at the origin (see Gyo91, Lemmas 2.5.3-2.5.4]). By Proposition 7.2 and flatness of $\mathscr{O}_{V}$ over $\operatorname{Sym}\left(V^{*}\right)$, Koszul freeness of a linear free divisor $D$ is equivalent to Koszul freeness of $(f, 0)$. By the same argument, strong Koszul freeness of $D$ is equivalent to condition (c) in Theorem 1.3 for $f_{0}$. Therefore, the claim follows by combining Theorem 3.5 and Proposition 7.4 (c) with Theorems 1.2 and 1.3 .

\section{Acknowledgments}

The second author wishes to thank David Mond for his comments on the first draft version of this article, and Michel and Jenifer Granger and the Université d'Angers for their hospitality in Summer 2008. We are grateful to Luis Narváez Macarro for pointing out Remark 7.3 .

The first author was partially supported by the ANR Sediga, Programme blanc $\mathrm{N}^{\circ}$ ANR-08-BLAN-0317-01/02. The second author was supported by the College of Arts \& Sciences at Oklahoma State University through a FY 2009 Dean's Incentive Grant.

\section{References}

[Ber72] I. N. Bernšteı̆n, Analytic continuation of generalized functions with respect to a parameter, Funkcional. Anal. i Priložen. 6 (1972), no. 4, 26-40 (in Russian). Zbl 0282.46038 MR 0320735

[Bjö79] J.-E. Björk, Rings of differential operators, North-Holland Math. Library 21, NorthHolland, Amsterdam, 1979. Zbl 0499.13009 MR 0549189

[BM06] R.-O. Buchweitz and D. Mond, Linear free divisors and quiver representations, in Singularities and computer algebra, London Math. Soc. Lecture Note Ser. 324, Cambridge Univ. Press, Cambridge, 2006, 41-77. [Zbl 1101.14013 MR 2228227

[CNM96] F. J. Castro-Jiménez, L. Narváez-Macarro, and D. Mond, Cohomology of the complement of a free divisor, Trans. Amer. Math. Soc. 348 (1996), 3037-3049. Zbl 0862.32021 MR 1363009

[CU04] F. J. Castro-Jiménez and J. M. Ucha-Enríquez, Testing the logarithmic comparison theorem for free divisors, Experiment. Math. 13 (2004), 441-449. Zbl 1071.14024 MR 2118269 
[CMNCJ02] F. J. Calderón Moreno, D. Mond, L. Narváez Macarro, and F. J. Castro Jiménez, Logarithmic cohomology of the complement of a plane curve, Comment. Math. Helv. 77 (2002), 24-38. Zbl 1010.32016 MR 1898392

[CN02] F. Calderón-Moreno and L. Narváez-Macarro, The module $\mathscr{D} f^{s}$ for locally quasihomogeneous free divisors, Compos. Math. 134 (2002), 59-74. Zbl 1017.32023 MR 1931962

[CN05] _ Dualité et comparaison sur les complexes de de Rham logarithmiques par rapport aux diviseurs libres, Ann. Inst. Fourier (Grenoble) 55 (2005), 47-75. Zbl 1089.32003 MR 2141288

[CN09] On the logarithmic comparison theorem for integrable logarithmic connections, Proc. London Math. Soc. (3) 98 (2009), 585-606. MR 2500865

[DD90] P. Deligne and A. Dimca, Filtrations de Hodge et par l'ordre du pôle pour les hypersurfaces singulières, Ann. Sci. École Norm. Sup. (4) 23 (1990), 645-656. Zbl 0743.14028 MR 1072821

[dGMS09] I. de Gregorio, D. Mond, and Ch. Sevenheck, Linear free divisors and Frobenius manifolds, Compos. Math. 145 (2009), 1305-1350. Zbl pre05625831| MR 2551998

[Dim91] A. Dimca, On the de Rham cohomology of a hypersurface complement, Amer. J. Math. 113 (1991), 763-771. Zbl 0743.14029| MR 1118460

[GMNS09] M. Granger, D. Mond, A. Nieto-Reyes, and M. Schulze, Linear free divisors and the global logarithmic comparison theorem, Ann. Inst. Fourier (Grenoble) 59 (2009), 811-850. Zbl 1163.32014 MR 2521436

[GS06] M. Granger and M. Schulze, On the formal structure of logarithmic vector fields, Compos. Math. 142 (2006), 765-778. Zbl 1096.32016 MR 2231201

[Gri69] Ph. A. Griffiths, On the periods of certain rational integrals. I, II, Ann. of Math. (2) 90 (1969), 460-495, 496-541. Zbl 0215.08103 MR 0260733

[Gro66] A. Grothendieck, On the de Rham cohomology of algebraic varieties, Inst. Hautes Études Sci. Publ. Math. 1966, no. 29, 95-103. Zbl 0145.17602 MR 0199194

[Gyo91] A. Gyoja, Theory of prehomogeneous vector spaces without regularity condition, Publ. Res. Inst. Math. Sci. 27 (1991), 861-922. Zbl 0773.14025 MR 1145669

[Har77] R. Hartshorne, Algebraic geometry, Grad. Texts in Math. 52, Springer, New York, 1977. Zbl 0531.14001 MR 0463157

[HM98] M. P. Holland and D. Mond, Logarithmic differential forms and the cohomology of the complement of a divisor, Math. Scand. 83 (1998), 235-254. Zbl 0941.32027 MR 1673922

[Hum75] J. E. Humphreys, Linear algebraic groups, Grad. Texts in Math. 21, Springer, New York, 1975. Zbl 0325.20039 MR 0396773

[Jac79] N. Jacobson, Lie algebras, Dover Publ., New York, 1979. Republication of the 1962 original. Zbl 0121.27504 MR 0143793

[Kar91] Y. Karpishpan, Pole order filtration on the cohomology of algebraic links, Compos. Math. 78 (1991), 213-226. Zbl 0743.32028 MR 1104788

[Kas77] M. Kashiwara, $B$-functions and holonomic systems. Rationality of roots of $B$ functions, Invent. Math. 38 (1976/77), 33-53. Zbl 0354.35082 MR 0430304

[Kim03] T. Kimura, Introduction to prehomogeneous vector spaces, Transl. Math. Monogr. 215, Amer. Math. Soc., Providence, RI, 2003. Zbl 1035.11060 MR 1944442

[M2] D. R. Grayson and M. E. Stillman, Macaulay 2, a software system for research in algebraic geometry, http://www.math.uiuc.edu/Macaulay2/

[Meb89] Z. Mebkhout, Le formalisme des six opérations de Grothendieck pour les $\mathscr{D}_{X}-$ modules cohérents, Travaux en Cours 35, Hermann, Paris, 1989. Zbl 0686.14020 MR 1008245 
[NM08] L. Narváez Macarro, Linearity conditions on the Jacobian ideal and logarithmicmeromorphic comparison for free divisors, in Singularities I, Contemp. Math. 474, Amer. Math. Soc., Providence, RI, 2008, 245-269. Zbl 1166.32006 MR 2454351

[Sai71] K. Saito, Quasihomogene isolierte Singularitäten von Hyperflächen, Invent. Math. 14 (1971), 123-142. Zbl 0224.32011 MR 0294699

[Sai80] Theory of logarithmic differential forms and logarithmic vector fields, J. Fac. Sci. Univ. Tokyo Sect. IA Math. 27 (1980), 265-291. Zbl 0496.32007 MR 0586450

[SKKŌ81] M. Sato, M. Kashiwara, T. Kimura, and T. Ōshima, Microlocal analysis of prehomogeneous vector spaces, Invent. Math. 62 (1980/81), 117-179. Zbl 0456.58034 MR 0595585

[Sch07] M. Schulze, Logarithmic comparison theorem versus Gauss-Manin system for isolated singularities, Adv. Geom., to appear.

[Ser01] J.-P. Serre, Complex semisimple Lie algebras, Springer Monogr. Math., Springer, Berlin, 2001. Zbl 1058.17005 MR 1808366

[Ter78] H. Terao, Forms with logarithmic pole and the filtration by the order of the pole, in Proceedings of the International Symposium on Algebraic Geometry (Kyoto, 1977), Kinokuniya Book Store, Tokyo, 1978, 673-685. Zbl 0429.32015 MR 0578880

[Tor04] T. Torrelli, On meromorphic functions defined by a differential system of order 1 , Bull. Soc. Math. France 132 (2004), 591-612. Zbl 1080.32011 MR 2131905

[Tor07] _ Logarithmic comparison theorem and $\mathscr{D}$-modules: an overview, in Singularity theory, World Sci., Hackensack, NJ, 2007, 995-1009. Zbl 1121.32005 MR 2342946

[WY97] J. Wiens and S. Yuzvinsky, De Rham cohomology of logarithmic forms on arrangements of hyperplanes, Trans. Amer. Math. Soc. 349 (1997), 1653-1662. Zbl 0948.52014 MR 1407505 